\title{
Introduction
}

\section{The Early Detection Research Network: A platform for communication and collaboration}

\author{
Lora Kutkat and Sudhir Srivastava* \\ Cancer Biomarkers Research Group, National Cancer \\ Institute, National Institutes of Health, Rockville, MD, \\ USA
}

Since the conception of the National Cancer Act nearly 30 years ago, extraordinary advances in cancer research have helped decrease the number of lives lost to cancer. Remarkable technology developments and novel cellular discoveries have broadened our understanding of cancer - an understanding that has helped improve how and when cancer is detected. Using sensitive technologies to unveil subtle changes in a cell's molecular circuitry, scientists are using these molecular changes as signposts along the path to cancer. Scientists and clinicians are assessing if changes that preclude a cell's transformation to cancer can be used in a practical way to identify individuals with early stage cancer or who are at risk for developing cancer.

One of the most important effectors of the clinical outcome of cancer is whether it is detected at an early stage. The use of sensitive technologies and a better understanding of cancer are helping scientists and clinicians do just that - detect early stage disease. In the beginning stages of development, precancerous and cancerous lesions are localized, and the tumor is most amenable to eradication. Tools to find cancer at such an early stage would then offer a unique window of opportunity during which intervention could prevent progression to widespread and perhaps fatal disease.

Improvements in cancer detection research and subsequent reductions in cancer mortality have been realized through increased emphasis on cancer prevention

*Correspondence to: Sudhir Srivastava, Ph.D., MPH, Chief, Cancer Biomarkers Research Group, Division of Cancer Prevention, National Cancer Institute, 6130 Executive Boulevard, Suite 330F, Rockville, MD 20852, USA. Tel.: +1 301496 3983; Fax: +1 301 402 8990; E-mail: ss1a@nih.gov. research. Among the highest priorities of the National Cancer Institute is the support of research to improve the health of the public by decreasing the incidence, mortality, and morbidity of cancer. The National Cancer Institute's Division of Cancer Prevention is fulfilling this mission by engaging basic and clinical scientists, epidemiologists, and statisticians in a search for, and validation of, early cancer biomarkers.

At the forefront of advances in prevention science and biomarker research is the Early Detection Research Network (http://cancer.gov/edrn), a collaborative effort supported by the Division of Cancer Prevention. The Network was assembled into a four-component, scientific consortium from the recommendations of the National Cancer Institute's the Early Detection Implementation Group in 1998. The careful design of the Network promotes the development and validation of biomarkers for early cancer and risk assessment by providing a unique model for collaborative research. This Network has the distinction of pioneering this approach to biomarker research and is the only forum of its kind for biomarker development and validation.

The annual workshop sponsored by the Early Detection Research Network provides a forum for interaction and collaboration between leading cancer researchers from around the world. Investigators and liaisons in this consortium meet regularly to discuss how to better decipher the molecular circuitry of cells and apply this understanding to the earlier detection of cancer. Using both the unconventional approach to biomarker research through scientific consortia and facilitating the regular interaction of leading experts, the Network is pursing biomarker development and validation at an unprecedented pace.

At the first scientific workshop organized by the Network in September 2000, Network investigators presented scientific research findings that detailed the latest advances and emerging issues in biomarker research. The two-day meeting addressed six session topics, including Advances in Organ Specific Molecu- 
lar Detection, Correlative Clinical Studies, Computational Analysis and Statistics, Informatics, Emerging Issues and Technology for Biomarkers of Risk, and Technologies within the Network. More than a dozen speakers and over 60 abstracts fostered discussions on these issues and other key challenges of the Network. The abstracts presented in this issue of Disease Markers document the research highlighted and presented at the first scientific conference of the Early Detection Research Network.

Through regular collaborations facilitated by the Network, investigators can face the challenges of biomarker research in a venue that facilitates communication and intellectual exchange. Challenges of creating novel approaches to validation studies, improving informatics and information flow, standardizing data reporting, generating statistical and computational tools, and standardizing reagents and assays complicate the development and validation of biomarkers for early cancer and risk assessment. Through the merged ex- pertise of basic biologists, clinicians, epidemiologists, and statisticians, Network investigators are developing solutions to the barriers that plague biomarker research.

Substantial headway in biomarker development has been made within the first year of the Network's operation, and the rapid progress documented in these published abstracts is a testament to the collaborative approach to research. Barriers are being overcome, collaborations are being forged, and a better understanding the molecular circuitry of cancer cells is being pursued. Previously unknown puzzle pieces that once thwarted attempts to reduce mortality from cancer are falling into place, and the Early Detection Research Network is well poised to find additional missing pieces. The Network provides both an opportunity and a challenge for the scientific community - an opportunity to make science work for people and a challenge to make this pioneering model of collaboration a productive scientific construct that others will emulate. 


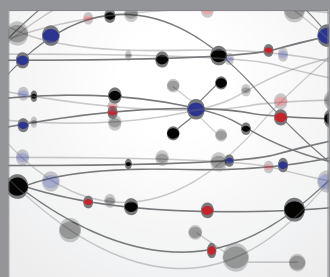

The Scientific World Journal
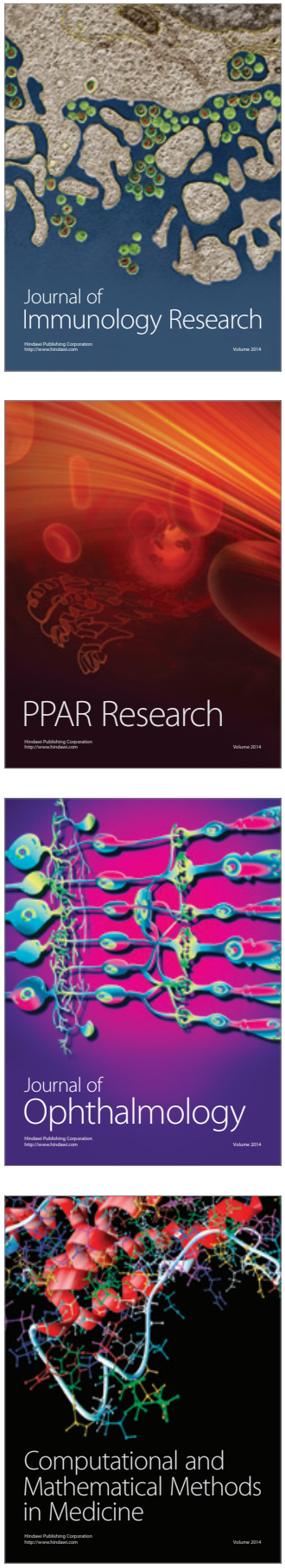

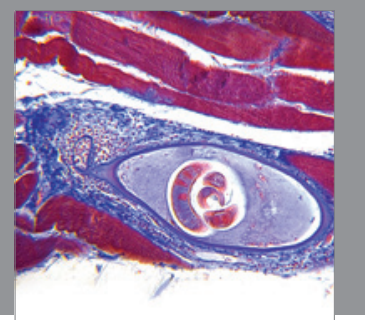

Gastroenterology

Research and Practice
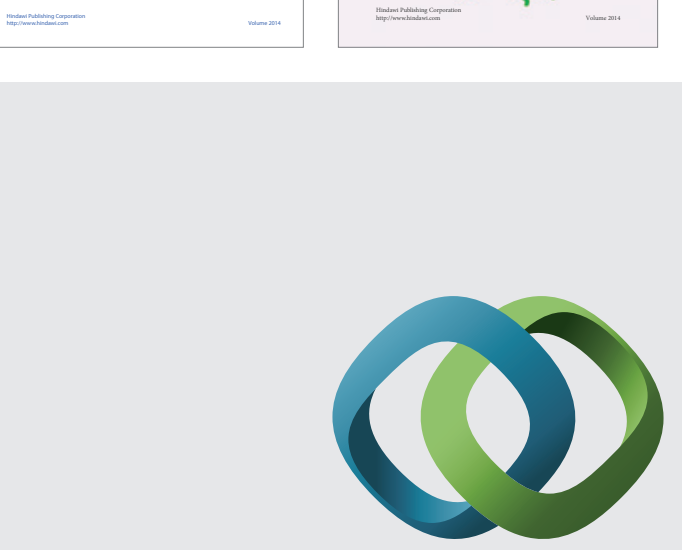

\section{Hindawi}

Submit your manuscripts at

http://www.hindawi.com
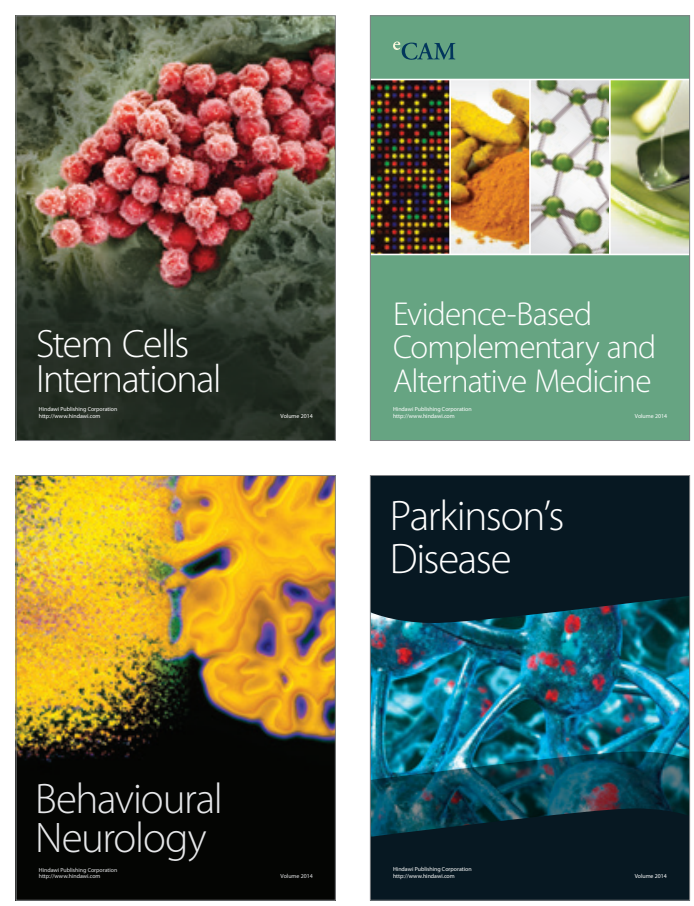

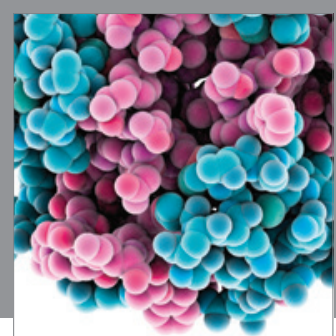

Journal of
Diabetes Research

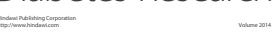

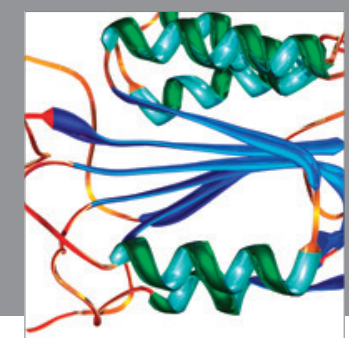

Disease Markers
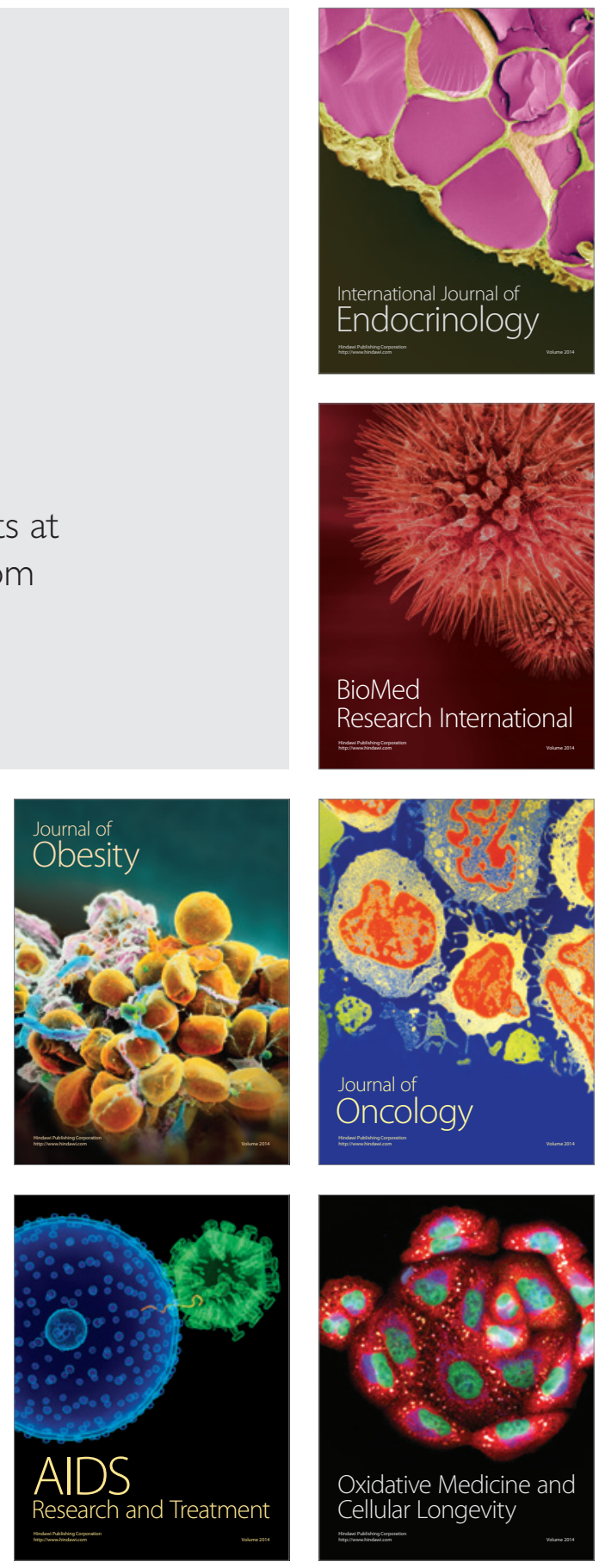